\title{
Generating apparent haptic motion for assistive devices
}

\author{
Inès Lacôte ${ }^{1}$, Claudio Pacchierotti ${ }^{2}$, Marie Babel $^{1}$, Maud Marchal ${ }^{1,3}$, and David Gueorguiev ${ }^{4}$
}

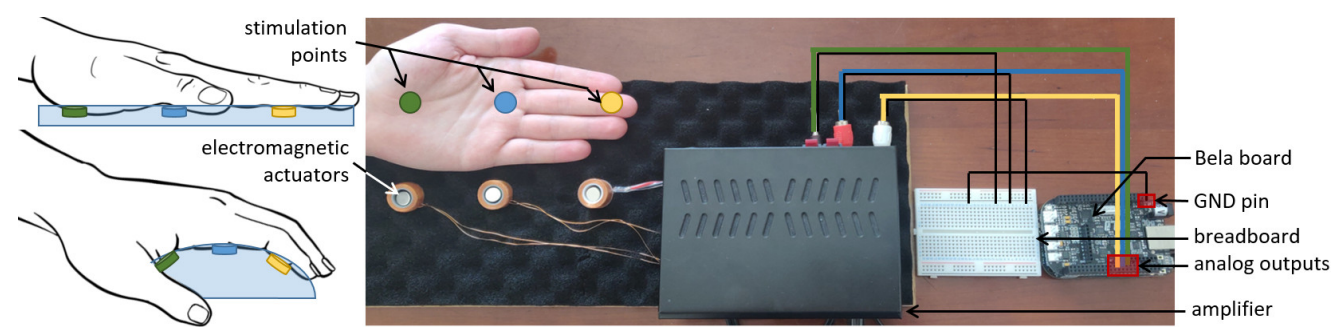

Fig. 1: Experiment setup on (left) flat and curved surfaces, generating stimuli on (right) three different locations on the hand.

\section{INTRODUCTION}

For people with mobility deficiencies, assistive technologies are sometimes not efficient enough to enable safe and independent navigation tasks. A way to alleviate this issue is to design haptic interfaces that can provide directional cues to the user during navigation. One promising cue is apparent haptic motion (AHM) in which a movement is perceived when a succession of discrete stimulations is actually delivered [1]. Often compared to analog illusions of visual and auditory senses [2], haptic illusions provide a wide range of possibilities to convey new sensations and make haptic interfaces more informative. Until now, mostly vibrations have been studied when it comes to AHM [3] and assistive technologies [4]. Besides, a large part of the contributions that can be found on this topic are performed on flat surfaces [5]. However, vibrations can engender neuromuscular fatigue, while the integration of this technology onto medical assistive devices such as walkers and powered wheelchairs requires to understand whether this illusion also holds when the resting surface is highly curved. This work aims to compare and quantify the difference in AHM perception for a vibratory mode and a continuous mode in which constant pressure is applied to the skin, both on flat and curved supports. Fig. 1 presents one of the tested curvatures; curvatures are tested until "closed hand position". The final goal is to determine whether pressure stimuli are a robust and efficient alternative to vibration in AHM.

\section{Material And Methods}

For this work, the apparent motion is generated along the palm and the middle finger using 3 custom designed electromagnetic actuators with a coil as a stator and a $10 \mathrm{~mm}$ diameter and $3 \mathrm{~mm}$-height magnet as a mover. A thin layer of silicon on the top of the actuators enables a soft contact with the hand placed on an adaptive surface with increasing curvature. To compare the vibratory mode and the continuous

\footnotetext{
This work was funded by Inria, under the challenge "DORNELL".

1 Univ Rennes, Inria, INSA, IRISA, CNRS - Rennes, France

2 CNRS, Univ Rennes, Inria, IRISA - Rennes, France

3 Institut Universitaire de France (IUF) - Paris, France

${ }^{4}$ CNRS, Sorbonne Université, ISIR - Paris, France
}

touch mode for AHM, two experiments are conducted with the same actuators using first a sinusoidal carrier within a triangular envelope and then a simple triangular signal without oscillations. These signals are sent at various speeds and intensities to the motors from 3 analog outputs on a Bela board created in the Centre for Digital Music at Queen Mary Univ., London, UK and then amplified by an operational amplifier (e.g., OPA544). The vibratory mode is realized using a frequency of $200 \mathrm{~Hz}$ and the continuous contact mode, pushing the magnet to raise in up then down-position into the coil, following a ramp signal. A recap of the set-up is shown in Fig. 1.

\section{HYPOTHESES FOR RESULTS AND CONCLUSION}

The actuators are functioning in both modes (vibratory and continuous) and a study of the two questions (comparison of vibratory vs. continuous modes; impact of the surface curvature) is planned in the coming month. The experiments will provide an insight whether vibrotactile stimuli are a main condition for conveying the apparent haptic motion and whether curvature can be increased while preserving the illusion. An interesting conclusion, if the obtained results present a similar efficiency in vibratory and continuous contact mode, would be that new devices for long period usages could be designed with other types of motors than vibrotactile actuators in order to lower the cognitive load and fatigue. Secondly, conclusions on impact of the curvature would permit to think of new designs for portable assistive haptic interfaces carrying handles for example.

\section{REFERENCES}

[1] S. Lederman and L. Jones, "Tactile and Haptic Illusions." IEEE Transactions on Haptics, vol. 4, no. 4, pp. 273-294, 2011.

[2] S. Lakatos and R. N. Shepard, "Constraints Common to Apparent Motion in Visual, Tactile, and Auditory Space," J Exp Psychol Hum PerceptPerform, vol. 23, no. 4, pp. 1050-1060, 1997.

[3] J. Park et al., "Rendering Moving Tactile Stroke on the Palm Using a Sparse 2D Array," in Proc. of Europhaptics. Springer, 2016.

[4] S. Gallo et al., "Augmented White Cane with Multimodal Haptic Feedback," in Proc. of IEEE RAS/EMBS BioRob, 2010, pp. 149-155.

[5] J. Seo and S. Choi, "Edge Flows: Improving Information Transmission in Mobile Devices using Two-dimensional Vibrotactile Flows," in Proc. of IEEE WHC, 2015, pp. 25-30. 